Harold A. McAlister and William I. Hartkopf (eds.)

\title{
Prospects for Rapid, Routine Speckle Photometry
}

\author{
D. J. BARRY ${ }^{1}$, W. G. BAGNUOLO ${ }^{1}$, B. D. MASON ${ }^{1}$, \\ H. A. MCALISTER ${ }^{1}, \&$ N. H. TURNER ${ }^{1}$ \\ Center for High Angular Resolution Astronomy, \\ Georgia State University, Atlanta, Georgia 30303
}

\section{INTRODUCTION}

Image reconstruction techniques have rapidly matured over the last decade, and increasing reference to full-aperture and masked aperture results appear in the literature. Although anomalies in morphology are understood, most work uses nonlinear iterative refinements of questionable photometric robustness. Recent development of linear least-squares phase reconstruction techniques from the bispectrum (Glindemann et al. 1991) offers hope for a robust technique using a computational tour de force. However, the field cannot be considered mature until routine photometry of a large number of speckle-resolved objects is performed and demonstrated to be repeatable. We review our work and our expectations for use of both computationally intensive and simple techniques in our routine speckle reductions.

\section{DATA QUANTITY AND SEEING}

Routine reduction is as much a problem of data storage and archiving as comparative analysis. The CHARA speckle archives contain over 5.8 terabytes (90 million frames, 40,000 observations)!

The Directed Vector Autocorrelation (DVA) algorithm, our current performance champ, yields primarily astrometric information with a quadrant identification, and costs $1 \mathrm{CPU}$ hour/video minute (MIPS 3000, $33 \mathrm{MHz}$ ). A complete re-analysis of the archives would require $5.7 \mathrm{CPU}$ years. It is possible that crude photometry will eventually be possible from existing DVA correlograms at low CPU cost (Bagnuolo et al. 1992).

The assumption of stable seeing, basis of power spectrum subtraction of nearby matched single stars, is a weak link in most reductions. We have found that seeing varies over timescales of a few seconds (cf. Figure 1), and that it is sensitive to dome azimuth. We have also made a preliminary unpublished observation that seeing at the CTIO 4-m can vary during moderate, 15-20 knot winds, as observed by monitoring the image profile of a zenithal star while rotating the dome through a complete rotation. Model subtraction is more unattractive than data subtraction, but has the advantage of speed and "reproducibility".

\footnotetext{
${ }^{1}$ Visiting Astronomer, Kitt Peak National Observatory, National Optical Astronomy Observatories, operated by the Association of Universities for Research in Astronomy, Inc., under contract with the National Science Foundation.
} 


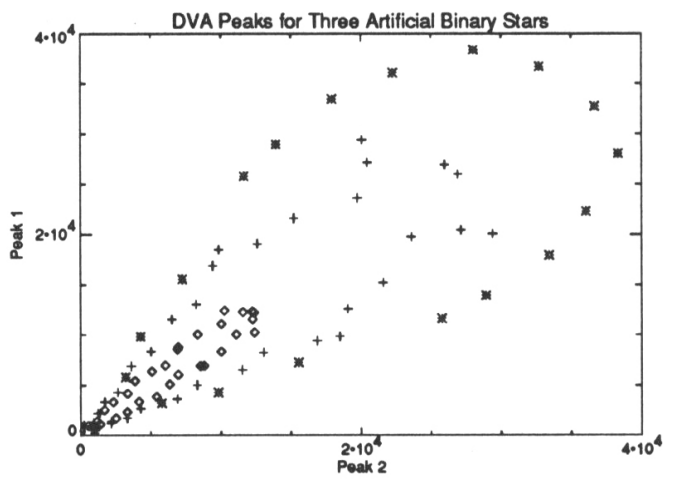

FIGURE 1. Peak intensity for a distribution of $\Delta M$ values is shown in the case of three total intensities, as generated by a calcite-polarizer combination. Asterisks are from $\zeta$ Persei, $M_{v}=2.85$. Plus signs are 58 Persei, $M_{v}=4.25$, and diamonds are HR 1712, $M_{v}=6.0$. As photon noise increases, the maximum peak ratio decreases, making $\Delta M$ discrimination more difficult.

\section{REDUCTION AND CALIBRATION}

The Fork Algorithm, a rapid, image-space method (Bagnuolo 1988), degrades non-gracefully with shot noise (cf. Figure 2). Thus, we have analyzed a number of systems from this method, but we have only published detailed analyses of Capella (Bagnuolo et al. 1988). The recent eclipse of $\gamma$ Persei offers the rare opportunity to check the accuracy of our unpublished Fork reductions of this star and to confirm the preliminary photometry for 51 Tauri and other stars in Dombrowski (1990). We present these preliminary Fork and DVA photometric results because of the possible extension of these methods to wholesale reduction of speckle data. It is difficult to imagine bispectrum techniques run on thousands of observations with existing affordable CPU/storage technology.

We are currently using a calcite crystal inserted into the optical path of our speckle camera to simulate a perfectly isoplanatic binary, where the $\Delta M$ is

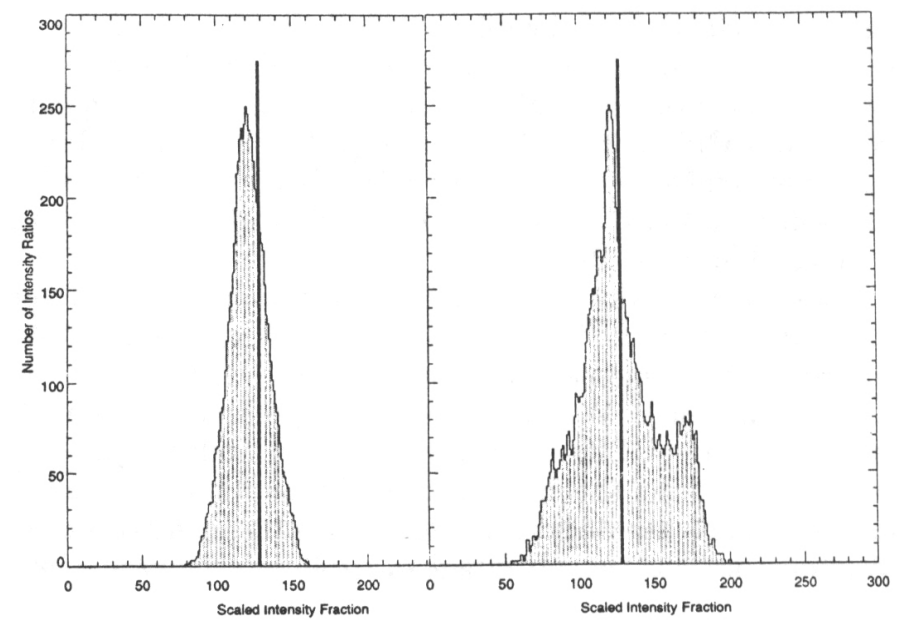

FIGURE 2. Two fork histograms showing noise degradation (from Dombrowski 1990). The leftmost image is a clean histogram of 51 Tauri. The rightmost, of Finsen 342 , showing a systematic noise background of unknown shape, illustrates the difficulty of extracting accurate values for dim stars by this technique. 
varied by a rotatable polarizer which modulates the ordinary and extraordinary beams of the crystal. With this artificial data, we have begun to calibrate our DVA method (cf. Figure 3) for crude "first look" photometry, with the goal of publishing columnar values for each of our observations.

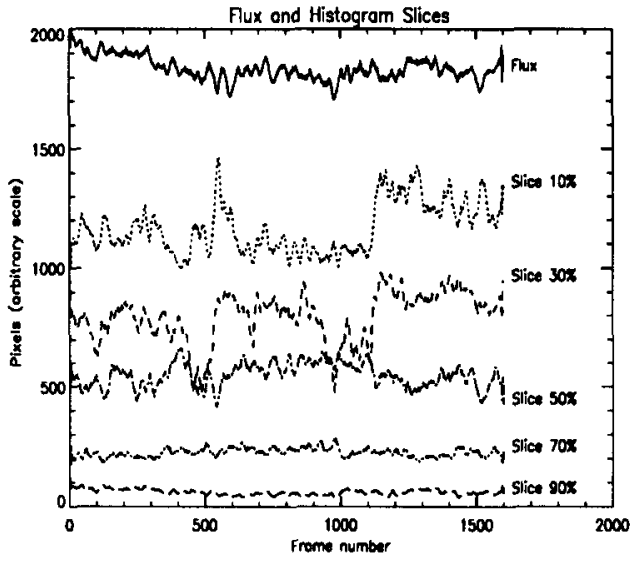

FIGURE 3. Intensity sum as a function of time (solid line), showing small variations from overflow of speckles outside the frame. Hatched lines are time-slices through a single abscissa point of brightness histograms: upper lines show number of low-intensity pixels, and are thus correlated with poor seeing. Lower lines show number of high-intensity (large $R_{0}$ ) pixels, and correlate with good seeing. Upper lines are seen to be anticorrelated to lower lines, and intensity dips are seen to be correlated with poor seeing (from greater speckle overflow).

\section{PROSPECTS REVISITED}

We believe that the current work of Glindemann et al. is the best tour de force method for use on important systems, although like all such techniques, its accuracy and precision remains unproven. We hope our routine use of the newest techniques on a variety of important systems will establish the limits of their applicability. Accurate $\Delta M$ information has the potential to close the loop on joint spectroscopic-visual orbit determinations, permitting most primary astrophysical parameters to be known for the single components.

We appreciate the support of NSF grant AST 89-15324, CIF funding of the Georgia Board of Regents, and the GSU College of Arts and Sciences.

\section{REFERENCES}

Bagnuolo, W.G. 1988, Optics Letters, 13, 907

Bagnuolo, W.G., Mason, B.D., Barry, D.J., Hartkopf, W.I., \& McAlister, H.A. 1992, $A J, 103,1399$

Bagnuolo, W.G. \& Sowell, J.R. 1988, $A J, 96,1056$

Dombrowski, E.G. 1990, Ph.D. thesis, Georgia State University

Glindemann, A., Lane, R.G., \& Dainty, J.C. 1991, "Bispectral Parameter Estimation using Least Squares", in press

Glindemann, A., Lane, R.G., \& Dainty, J.C. 1991, "Estimation of Binary Star Parameters by Model Fitting the Bispectrum Phasen, in press

Glindemann, A., Lane, R.G., Dainty, J.C. 1991, "Least Squares Reconstruction of the Object Phase from the Bispectrum", Digital Signal Processing-91, eds. V. Cappellini and A.G. Constantinides, (Elsevier Science Publishers) 